\title{
Publisher Correction: Determining T-cell specificity to understand and treat disease
}

Sine Reker Hadrup and Evan W. Newell

Correction to: Nature Biomedical Engineering 1, 784-795 (2017); published online 10 October 2017.

In the version of this Perspective originally published, in Fig. 4 , in the schematic for the DNA barcoded multimers, the barcodes were missing; they have now been included and the figure updated in all versions of the Perspective.

Published online: 2 January 2018

https://doi.org/10.1038/s41551-017-0176-8 